\title{
Concept and Consequences of the EU Biocide Guideline
}

\author{
H.-C. Flemming (『) and M. Greenhalgh
}

\begin{abstract}
The Biocide Product Directive (BPD) of the European Union is intended to balance the efficacy of biocides in their intended use with their impact on human and animal health and the environment. It legally organizes the process of putting a biocide on the market and harmonizes the regulation of the EU Member States. Biocides have to be subjected systematic tests for efficacy and risk before approval. The BPD achieves its aims using a two-stage regime of rigorous evaluation of biocidal active substances and products, to ensure they pose no unacceptable risks to people, animals or the environment.

Ultimately only those biocidal products that contain an active substance that is included in Annex I of the Directive will be authorized for use. Active substances have to be evaluated to ascertain whether or not they will be included in Annex I. This requires industry to submit data, which is evaluated by Member States with decisions over Annex I inclusion being taken at the European level. Industry is charged a fee for this process. Once an active substance has been included in Annex I, national Competent Authorities can authorize products containing it in individual Member States (providing that any necessary data have been supplied and any conditions put on Annex I inclusion are met). Once a product has been authorized in the first Member State, it will be possible for it to be mutually recognized and therefore authorized by other Member States (providing relevant conditions are similar). However, there will have to be an application from other Member States, and again there will be a fee for these processes.
\end{abstract}

\section{Brief Historical Outline}

By nature, biocidal products are directed against living organisms, frequently not really restricted to "target organisms". This implies that they inevitably also can harm the health of non-target organisms such as humans or animals. An example is DDT,

H.-C. Flemming

Biofilm Centre, University of Duisburg-Essen, Geibelstrasse 41, D-47057, Duisburg, Germany

e-mail: hanscurtflemming@compuserve.com 
which was very effective against mosquitoes spreading malaria but was bio-accumulated and spread into the environment to an extent that made the further use of this substance unacceptable. Furthermore, a wide variety of substances for biocidal use have been developed and applied worldwide. They are authorized by regulations that are very different in different countries, frequently incompatible, and based on (partially insufficient) systems of risk assessment. In order to overcome this situation, the European Commission drafted legislation for harmonizing provisions for biocidal products, and to ensure a more uniform and higher level of health and environmental protection throughout the EU without compromising the internal market. This was (and still is) a very challenging approach and the European Commission has gone further by co-operating with other non-EU countries through the auspices of the Organisation for Economic Co-operation and Development (OECD) to try to harmonize biocide regulations on a global basis. The basic idea is that it is not enough just to develop a new and more effective biocide but that the health and environmental issues involved in its application are also considered. The concept of the authors of the guideline was to implement a system that would force the chemical industry to behave in an ethically acceptable way. In 1993, the first draft of a Directive concerning the placing of biocidal products on the market was submitted by the European Commission. It was found that risk assessment should be an integral part of the Directive. The Commission therefore submitted a revised version in 1995. After long and controversial discussions, a final text was adopted by the Council in 1998 as "Biocidal Products Directive" (BPD) (European Parliament 1998). Each Member State appointed an agency to deal with the new legislation: the so-called Competent Authority. The biocides industry transformed dramatically with the implementation of the BPD. There has been much debate amongst industry and the national Competent Authorities on how to operate the scheme, within the idealistic legal framework of the Directive. Some Competent Authorities themselves are considered by some to take an idealistic approach, whereas others are more pragmatic and give higher priority to the needs of industry and biocide users. Nevertheless, the BPD is significantly more stringent than any previous legislation, either within Europe or in the rest of the world. Knight and Cooke (2002) assume that it may cost the industry well over 500 million $€$. Much of the information provided in this chapter is based on their excellent work.

From the point of view of health and environment, it clearly is a major step forward to better protection, although the direction is very complicated in many details and it is not free from discrepancies.

\section{Scope of the Guideline}

The BPD defines biocidal products as "preparations containing one or more active substances that are intended to control harmful organisms by either chemical or biological, but not physical, means". This encompasses a wide range of products including disinfectants, insect repellents, and anti-fouling paints. Despite the name "biocide", a biocidal product does not actually have to kill. If it is used to destroy, 
deter, make harmless, or control a harmful organism by chemical or biological means, it maybe considered to be a biocide. For example a repellent used to "deter" a mosquito could be considered to be a biocidal product.

The Directive will not apply to certain products already subject to European legislation, including plant protection products, human medicines, veterinary medicines, medical devices or cosmetics. Article 1 of the BPD lists those Directives that are not covered within the scope of the BPD. The legislation also excludes the nonbiocidal uses of products and active substances

The official objective of the guideline is laid down in the Foreword, Chap. 1, of the guideline:
"Whereas, in their resolution of 1 February 1993 on a Community programme of policy and action in relation to the environment and sustainable development $\left({ }^{4}\right)$, the Council and the representatives of the Governments of the Member States, meeting within the Council, approved the general approach and strategy of the programme presented by the Commission, in which the need for risk management of non-agricultural pesticides is emphasised;"

In Chap. 3 of the Foreword the EU commits that biocides are necessary for the control of organisms dangerous for the health of humans and animals:

\footnotetext{
"Whereas biocidal products are necessary for the control of organisms that are harmful to human or animal health and for the control of organisms that cause damage to natural or manufactured products; whereas biocidal products can pose risks to humans, animals and the environment in a variety of ways due to the intrinsic properties and associated use patterns;"
}

These are the official scientific reasons for implementing the BPD.

The biocidal products guideline is divided into three parts. The third and last one, the Annexes, is potentially the most important because it lists the conditions for placing biocidal products on the market. Annex V of the BPD classifies biocidal products into four main groups: disinfectants and general biocides, preservatives, pest controls, and other biocides as shown in Table 1 (Knight and Cooke 2002).

Ultimately only those biocidal products that contain an active substance that is included in Annex I of the Directive will be authorized for use. An area of dispute concerning scope is the regulation of in-situ generated biocides. These include substances that are mixed together or otherwise generated by the consumer to create the biocidal active ingredient. The EU Commission and Member States have agreed that for example the in-situ generation of ozone is not covered.

When the Directive has been fully implemented in all Member States, existing and new active substances will have to be evaluated to ascertain whether or not they will be included in Annex I. Both processes will require industry to submit data, with a system of data protection. The data will be evaluated by Member States with decisions over Annex I inclusion being taken at the European level. Industry will be charged a fee for this process.

Once an active substance has been included in Annex I, national Competent Authorities can authorize products containing within individual Member States (providing that any necessary data have been supplied and any conditions put on Annex I inclusion are met). 
Table 1 Products defined as biocides within the BPD

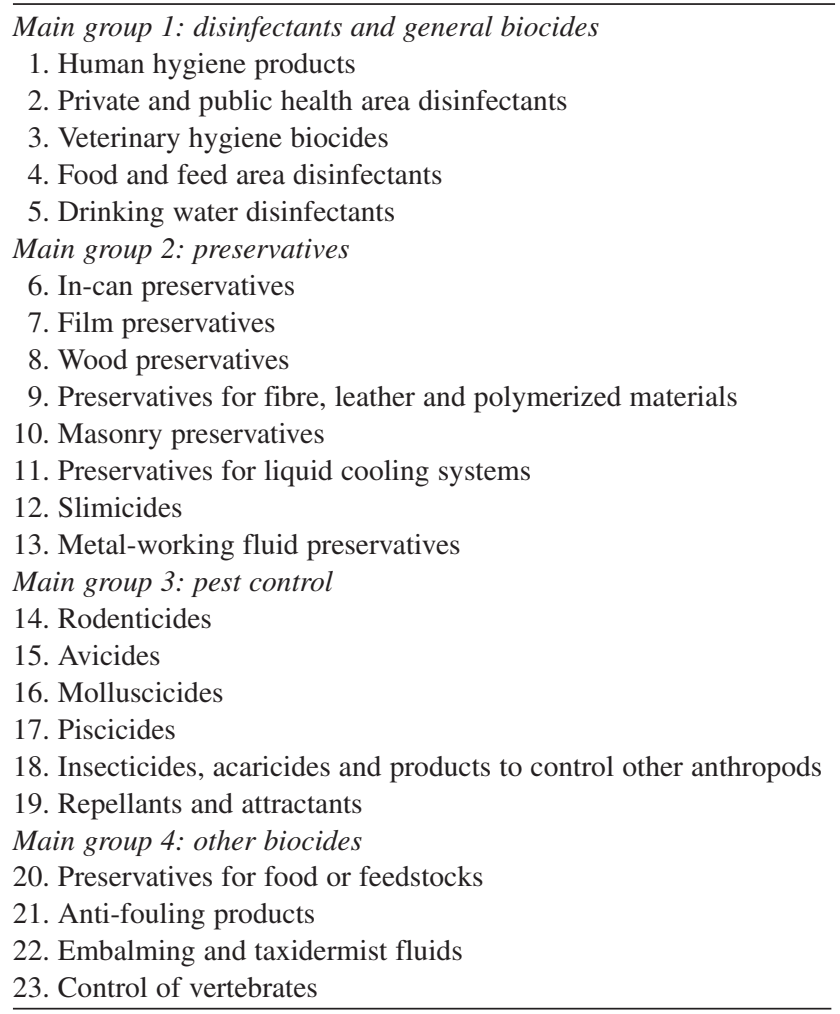

Following product authorization in a first Member State, it is then possible for the product to be mutually recognized and therefore authorized by other Member States (providing relevant conditions are similar). However, there will have to be an application to other Member States, and once again there will be a fee for these processes.

Each European Union Member State is responsible for implementing the BPD. In Great Britain the Directive was implemented through the Biocidal Products Regulations 2001, which came into force on 6 April 2001. The Directive was implemented in Northern Ireland through the Biocidal Products Regulations (Northern Ireland) 2001 on 16 January 2002. The Biocidal Products (Amendment) Regulations came into force on 1 April 2003 and there has been a further amendment, the Biocidal Products (Amendment) Regulations 2005, which came into force on 1 October 2005.

\section{Approval Systems}

To obtain authorization for the marketing of a biocide, the applicant must submit at least two data packages: the first on the active substance, and the second on the formulated product relating to the product type. For each additional product type 
for which authorization is sought, a further dossier is required at additional cost. The BPD makes a pragmatic but arbitrary distinction between those biocidal active substances on the market before 14 May 2000 ("existing" active substances) and those placed on the market for the first time after this date ("new" active substances). The Member States and the Scientific Committee on Biocidal Products review the scientific content of the dossier and make an appropriate recommendation to the Commission. If the recommendation is favourable, the Commission will enter the active substance in an approved list (Annex I of the BPD). A review programme is established in the BPD to assess systematically during a 10-year period all the existing active substances (European Commission 2006).

Standard biocidal products containing active substances in Annex 1 of the BPD require a full dossier of information, and applications for authorization are evaluated by the national Competent Authority without undue delay. Biocidal products containing a new active substance for which a decision for Annex 1 listing is pending may be provisionally authorized for up to 3 years. New products containing existing active substances can be authorized under existing national schemes for up to 10 years during the review programme. Under the BPD, applicants may use the concept of "frame formulation" to facilitate authorization of re-branded biocidal products. Frame formulations are groups of biocidal products with the same active substance of the same technical specification and use, which differ only in minor details of the formulation composition such as colour or perfume ingredients, and hence have the same risk and efficacy. Once a Member State approves a biocidal product, all other Member States must approve the product, according to the principle of mutual recognition. If there are disputes between Member States by some reason, the Standing Committeeon Biocidal Products will have to resolve them. Member States may opt out of the mutual recognition procedure for avicide, piscicide and vermin-control biocidal products.

\section{Risk Assessment}

Risk assessment is an important aspect of the regulatory process. It is performed for both the intended use and a reasonable worst-case situation. The risk from a chemical substance is determined from its intrinsic hazardous properties and the likely exposures of humans and the environment throughout its life-cycle. The intrinsic chemical, health, and environmental hazardous properties can be quantified as a hazard assessment. The hazard of the biocide is assessed predominantly through toxicological testing in animal models (Annex IIA and IIB). Good quality human data may also be available, perhaps from epidemiological studies. The hazard assessment is combined with an exposure assessment to produce a risk assessment. If the outcome is favourable, the substance will be recommended for Annex 1 listing. If not, further information on toxicity or exposure in order to refine the risk assessment may be demanded. If the risk remains unfavourable, a regulatory decision may be taken to implement risk management requirements, such as additional labelling or restrictions to use, to permit product approval. 
Exposure assessment is a more complex issue. There are two basic options: measuring or modelling. Modelling can be carried out using generic data for chemical release. Estimates of environmental release are improved by gathering information on the release of biocides from specific processes to develop emission scenarios. Risk characterization is also conducted regarding animals kept and used by humans. The humaneness of biocidal products targeted at vertebrates is also considered, e.g. for biocides directed against rats.

The rule is that biocidal products can only be approved if, when used as prescribed, they do not present unacceptable risks to man, animals or the environment, are efficacious and use permitted active substances. Approval of biocidal products requires that they are used properly at an effective but minimized application rate. The regulatory authority also assesses the packaging, labelling and accompanying safety data sheet.

Acute and repeat-dose toxicity, irritation and corrosivity, sensitization, mutagenicity, carcinogenicity, toxicity for reproduction and the physicochemical properties of each active substance in the biocidal product are considered. If possible, they are also quantified, preferably as a dose-response effect. This includes the exposure of professionals, non-professionals, and those exposed indirectly via the environment to each active substance in the biocidal product during its lifestyle. Only as a last resort is the use of personal protective equipment taken into account to enable a biocidal product to be used safely. Replacement of hazardous substances by non-hazardous ones is preferred. Biocidal products containing category 1 or $2 \mathrm{~A}$ or $2 \mathrm{~B}$ carcinogens, mutagens or substances toxic to reproduction cannot be approved for use by the general public. Carcinogens are defined after the International Agency for Research on Cancer (1987):

Category 1 is for substances for which there is sufficient evidence for a causal relationship with cancer in humans (confirmed human carcinogen)

Category $2 A$ is for substances for which there is a lesser degree of evidence in humans but sufficient evidence in animal studies, or degrees of evidence considered appropriate to this category, e.g. unequivocal evidence of mutagenicity in mammalian cells (probable human carcinogen)

Category $2 B$ is for substances for which there is sufficient evidence in animal tests, or degrees of evidence considered appropriate to this category (possible human carcinogen)

\section{Costs for Authorization of a Biocidal Product}

The Health and Safety Executive of the United Kingdom (2008) gives an interesting look at the current costs for authorization of a biocidal product, which is available from its website (http://www.hse.gov.uk/biocides/index.htm).

For Annex I inclusion the HSE currently charges $£ 10,000$ (approximately US\$ 20,000 ) for a completeness check of a dossier prior to full evaluation, in addition to an evaluation fee of $£ 84,000-89,000$ for the full evaluation. The fees are based on 
actuals, meaning that if it was calculated that more time was spent on an evaluation, a further fee to cover the work may be charged; consequently if less time was spent on an evaluation the calculated difference could be refunded.

The UK figures quoted here, are estimates of the likely costs involved in evaluating a product dossier. At the time of writing there are three active agents authorized in Annex 1, i.e. dichlofluanid, difethialone and sulphuryl fluoride, while carbon dioxide has been authorized in Annex 1A. Actual costs are unknown, but costs for product authorizations will vary depending on how relevant the data (that was supplied for inclusion into Annex I of the BPD) is to a product. In the UK, the estimate for the first product authorization after Annex inclusion could cost between $£ 8,500$ and $£ 20,000$. The fee will be based on the actual work done and will depend on how much of the work was done at the Annex I inclusion stage. Once the initial product has been authorized, fees will be lower. It is expected that products can be authorized that contain the same active ingredient and are the same formulation etc. as the original product, providing the company seeking authorization holds the relevant letters of access. Also it is expected that the cost for authorization of subsequent products that have the same use, user type and contain the same active substance, but with differences in composition from a previously authorized product that do not affect the level of risk or efficacy associated with the product, is to be in the region of $£ 500$.

\section{Some Problems with the BPD}

The given definition for biocides as given in the BPD is very broad. It includes chemical compounds, formulations of compounds and also microoganisms and viruses, which strictly are not chemical substances. The entire legal work is not without inconsistencies and discrepancies. Thus, organisms such as Bacillus thuringiensis are dealt within a guideline that was created to reduce the usage of hazardous substances such as the "Seveso toxin" dioxin (2,3,7,8-tetrachlordibenzo- $p$-dioxin and 2,3,7,8-tetrachlor-dibenzofuran).

Even substances that are commonly accepted as non-toxic are enclosed into this definition: Ethanol for instance is not poisonous according to the guideline 67/EWG. However, if a technical product (e.g. a cleaner) contains sufficient amounts of ethanol, it will be preserved against microbiological growth just by that ethanol. So it is debatable whether ethanol is added to improve the cleaning results when applying the product or whether ethanol is added to inhibit microbiological growth.

The problem leads to the transfer of judicial power to the public administration. The administration may decide on the basis of the form sheet of the biocidal product whether an ingredient is in fact a biocidal substance or not. The administration uses the information provided when other users of biocidal products registered their active ingredients before. When the notification process is completed, the public administration will have had to deal with many more borderline cases like that outlined above. So, the public administration will have to build up the capability to 
evaluate biocidal compounds and biocidal products as well as decide which biocidal compound or which biocidal product may be placed on the market and which may not. Thus, the biocidal product guideline 98/8/EG not only imposes a heavy burden on the chemical industry but also on the public administration simply because of the immense administrative time and effort. The process of implementation is designed in a way that the public authorities must learn and understand how biocides act in a scientific way as well as the benefits they give to the user and the damage they may potentially cause to human health and the environment. This all takes a considerable time to accomplish.

The BPD guideline differentiates between biocidal substances and biocidal products. Biocidal products are materials that are used to control harmful microorganisms or wildlife, such as rodents. Biocidal substances are chemical substances that have the ability to kill or inactivate target organisms and are used in biocidal products for control of microorganisms or wildlife.

Biocidal substances (active agents) have to be listed in Annex 1 to be allowed to be used in biocidal products. Biocidal substances that are not dangerous substances according to the guideline 67/EWG may be listed in Annex IA. To have a substance listed in Annex IA is more difficult than to have it listed in Annex 1, but the listing of a biocidal product that contains the substance is easier. Biocidal substances that are so common that their usage cannot be controlled by the administration will be listed in Annex IB. The guideline gives some examples for such substances. Quoted in the guideline itself are ethanol and carbon dioxide, used so widely that they are listed in Annex IB.

To be placed in Annex 1, IA or IB, the items laid down in Annex 2 have to be determined by tests and their results have to be disclosed to the public administration. To ensure that the results of the different tests from different laboratories are comparable, test methods have to be developed and rules for the interpretation of the test results have been published. A list of properties of the substances, which have to be determined for substances to be listed in one of the chapters of Annex I is given in Annex IIA. The Annex IA gives a list of data that have to be specified by test results or by other data of equivalent significance and reliability. A major weighting is placed upon data relating to the fate of the biocidal compound in the environment. Data have to be submitted on the toxicity against aquatic organisms, the fate of the compound in soil, in addition to data about the biological degradation. Biodegradation is especially important as biocidal compounds must not be allowed to build up in the environment. The types of tests are legally determined, for example OECD biodegradation study protocols. Some tests that have been undertaken to allow chemical compounds to be placed on the US market will have to be repeated as the US tests use different vertebrate species.

Annex IB lists the data necessary to place a biocidal product on the market, which consists of biocidal compounds plus additional substances that might influence the activity of the biocidal substance.

The type of data necessary to be allowed to place a compound or a product on the market depends on the intended use or the purpose of the biocidal compound or biocidal product. Annex V lists 23 different purposes for biocidal compounds or 
products (product types). By legal definition this list of purposes is considered to be complete so that all notified biocidal compounds or products have to be listed within one of the defined 23 product classes (see Table 1). The decision whether a biocidal substance may be placed on the market is made by one of the national public administrations of the Member States of the EU. Currently most substances that have been filed under the notification scheme have been on the market prior to the introduction of the biocidal product Directive. All existing biocidal substances (i.e. those placed on the market before 14 May 2000), which are to be notified and supported, have been placed onto four lists with dossier call-in dates of 28 March 2004, 30 April 2006, 31 July 2007 and 31 October 2008. Once dossiers are received the review process begins. The reviews have been distributed among the national public administrations (Competent Authorities) according to their capabilities. The amount of data that has to be controlled by the public administration to be able to decide whether a substance may be placed on the market is so large that the administrations have yet to finish their work. One important date was 1 September 2006, at which time all identified existing actives that were not notified as being supported through the authorization process must be withdrawn from the European Union.

Once a decision is taken by one of these national public administrations it has to be accepted by the other Member States, as long as there are no cogent scientific reasons against it. It could be for instance, that one Member State rejects the decision of the administration of another one because the target organism, which should be controlled by the compound, does not occur in the Member State, which then rejects the decision. A compound can be notified for a period of not more than 10 years for non-toxic substances and not more then 5 years for toxic substances. After that period the notification has to be renewed. If new data or new scientific knowledge becomes known to the administration that are in contrast to the data presented for the notification and make it desirable to withdraw the substance from the market for safety or environmental reasons, the public administration has to withdraw the notification and thus the substance from the market.

\section{Consequences of the Guideline}

In general, the BPD acts as a threshold for the development and diversification of the biocide market. This is intended to control developments that might otherwise lead to unwanted effects on health and the environment and takes responsibility for preventing such effects. It represents a real threshold because generating the data necessary to notify a compound or a product is very expensive and time-consuming. Estimates range from about 2-10 million $€$ (approximately US\$ 15 million) for testing and generation of the data in addition to the costs of the administration process. Therefore, only highly profitable companies are able to afford the time-consuming process and the costs required to notify a substance. The lower the data requirements, the lower the risk of losing money by the notification process due to 
insufficient sales after putting the compound or the product on the market. This doesn't take into account the additional time and costs associated with the invention and development of new active agents. Companies have tended to notify compounds and products that are have already been on the market for a long time and of which most of the data required by the administration have been generated and paid for already. Consequently, the system of notification implemented by the biocidal products guideline $98 / 8 / \mathrm{EC}$ is advantageous to old products. Given the market size for the various biocidal product types and the barrier of the BPD (including the substitution principle) most EU-based companies have withdrawn their new active research programmes.

Initially more than 1,500 active substances were identified, of which around 800 were notified to the EU Commission as being supported through the authorization process. Only approximately half of these dossiers have been submitted, leading to the conclusion that only a total of approximately 400 will go through the review process. As a consequence, the EU's Competent Authorities have had to rearrange their dossier review process.

Despite its complexity, the problems of internal coherence, and the political problems within the EU the BPD still represents a serious and responsible compromise to balance the intended effect of biocides (i.e. killing organisms) and the protection of humans and the environment against the non-intended effects. On the other hand, the BPD has certainly slowed down the development and implementation of new biocides.

Additional EU Directives such as the so-called VOC (volatile organic compounds) Directive (European Parliament 1999), and REACH (registration, evaluation, authorization and restriction of chemicals) (European Parliament 2006) have complicated the issue of biocidal products. The aim of the VOC Directive is to significantly reduce the use of VOCs within the EU. However, this has resulted in more water-based products, which will require preservation. With REACH, aspects of this Directive will impact on components of biocidal products. This Directive seeks to regulate a further 30,000 chemicals found within the EU. It is estimated that it will result in around 120,000 dossiers, something that will pose significant strain on both industry and national authorities.

For the development of new biocides, the BPD represents a significant barrier concerning the European market. This includes many chemical anti-fouling approaches, which have to be thought over on this background. It is a wake-up call against the unregulated dispersion of chemical agents, which tend not to be considered sufficiently. Therefore, alternative anti-fouling strategies such as nutrient limitation, cleaning-friendly design and surfaces gain a specific advantage over conventional chemical treatment.

\section{References}

Commission of the European Communities (2007) Commission Regulation (EC) no 1451/2007 of 4 December 2007 on the second phase of the 10-year work programme referred to in Article 
16(2) of Directive 98/8/EC of the European Parliament and of the Council concerning the placing of biocidal products on the market. Off J Eur Union L325:11.12.2007

European Parliament and the Council of the European Union (1998) Directive 98/8/EC of the European Parliament and of the Council of 16 February 1998 concerning the placing of biocidal products on the market. Off J Eur Commun L123:24.04.1998 http://ecb.jrc.it/ legislation/1998L0008EC.pdf. Last accessed 13 July 2008

European Parliament and the Council of the European Union (1999) Council Direcive 1999/13/ EC of 11 March 1999 on the limitation of emissions of volatile organic compounds due to the use of organic solvents in certain activities and installations. Off $J$ Eur Commun L85:29.3.1999

European Parliament and the Council of the European Union (2006) Regulation (EC) No 1907/2006 of the European Parliament and of the Council of 18 December 2006 concerning the registration, evaluation, authorisation and restriction of chemicals (REACH). Off J Eur Union L396:30.12.2006

Health and Safety Executive of the United Kingdom (2008) Biocides and pesticides. http://www. hse.gov.uk/biocides/index.htm. Last accessed 13 July 2008

International Agency for Research on Cancer, World Health Organisation (1994) IARC monographs on the evaluation of carcinogenic risks to humans, vols 1-60, 1972-1994 and Suppl 7, 1987

Knight, DJ, Cooke, M (2002) Regulatory control of biocides in Europe. In: Knight, DJ, Cooke, M (eds.) The biocide business. Wiley, Weinheim, pp. 45-74 